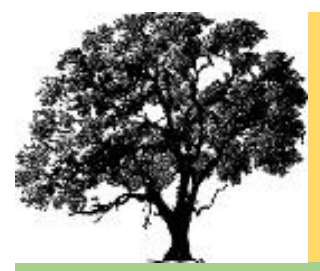

\title{
Sivas'ta doğal olarak yetişen bazı odunsu ve çalı türlerinin peyzaj mimarlığında kullanım olanaklarının belirlenmesi
}

\author{
Selvinaz Gülçin BOZKURT iD \\ Fenerbahçe Üniversitesi, Mühendislik ve Mimarlık Fakültesi, İç Mimarlık ve Çevre Tasarımı Bölümü, İstanbul- \\ Türkiye
}

Sorumlu Yazar: sbozkurt00@gmail.com

\section{Özet}

Dünyada kentleşmenin artması, açık ve yeşil alanların hızlı bir şekilde azalmasına neden olmaktadır. Buna paralel olarak bitkilerin, özellikle doğal türlerin peyzaj onarımında işlevsel katkılarının olması bitkilendirme çalışmalarındaki önemlerini hızla arttırmaktadır. Ekosistem hizmetlerinde işlevselliğin yanı sıra görsel özellikleri ile öne çıkan türlerden bazı odunsu ve çalı türleri gerek kentsel, gerekse de kırsal peyzaj çalışmalarında farklı kullanımları (farklı form, doku vb.) tercih edilmektedir. Sivas ili de geniş yeşil alanlara sahip olması nedeniyle birçok doğal bitki türüne ev sahipliği yapmaktadır. Bu nedenle, bu çalışma kapsamında Sivas’ta doğal olarak yetişen odunsu ve çalı türlerinin peyzaj mimarlığında kullanım olanakları incelenmiştir. Araştırma kapsamında öncelikle alanda doğal olarak yetişen odunsu ve çalı türleri belirlenmiş, bu türler arazide tespit edilerek peyzaj tasarımlarında kullanım olanakları irdelenmiştir. Bu çalışmalar sonucunda, alanda 21 familyaya ait toplam 56 takson peyzaj mimarlı̆ğ açısından fonksiyonel (erozyon önleme, perdeleme, karayolu kenarı ve refüj bitkilendirmesi, maden alanı yeniden ağaçlandırma, kaya bahçesi kullanımı, su kıyıları ve kıyı koruma) ve estetik özelliklerinin (çiçek güzelliği, yaprak güzelliği, meyve etkisi, form güzelliği, gövde güzelliği, gölge etkisi, sonbaharda yaprak renkliliği, kış etkisi) olduğu belirlenmiştir. Bu özelliklerinden dolayı alanda tespit edilen doğal odunsu ve çalı türlerinin; parklar, endüstriyel bölgeler, kamu ve özel bahçeler ile karayolu şev ve refüjlerinde soliter ya da gruplar halinde kullanılmaları önerilmiştir.

Anahtar Kelimeler: Bitkisel tasarım, Odunsu ve çalı türleri, Peyzaj mimarlı̆̆ı, doğal bitki türleri, Sivas

Determining the possibilities of using some woody and shrub species naturally grown in Sivas in landscape architecture

\begin{abstract}
Rapid urbanization in the world causes an important decrease in green spaces, that highlights the importance of natural plants day by day. Especially in landscape design, the use of plants for visual and functional purposes is gaining importance. Among these, some woody and shrub species are preferred to be used for design purposes in both urban and rural landscape design due to their functional characteristics as well as their flower, form, and fruit characteristics. Sivas province embraces many natural plant species due to its extensive green spaces. Therefore, within the scope of this study, the possibilities of using woody and shrub species naturally grown in Sivas were examined. Within the scope of the research, first of all, woody and shrub species that grow naturally in the area were determined by field trips and their usage in landscape designs were examined. As a result of this study, a total of 56 taxon from 21 families were found in the area. Two main characteristics in terms of landscape architecture were revealed as functional (erosion prevention, screening, highway side and median planting, mining
\end{abstract}


area reforestation, rock garden use, water shores and coastal protection) and aesthetic characteristics (flower beauty, leaf beauty), fruit effect, form beauty, stem beauty, shadow effect, leaf colour in autumn, winter effect). Due to these characteristics, this study suggested that the natural woody and shrub species detected in the area could be used solitarily or in groups in parks, industrial areas, public and private gardens, highways, and refuges.

Keywords: Plant design, Woody and shrub species, Landscape architecture, natural plant species, Sivas

\section{Giriş}

Günümüzde aşırı nüfus artışı, kentleşme ve endüstrileşme süreci ekosistemin bozulmasının en büyük nedenlerinden biridir. Doğal çevre üzerinde oluşan bu antropojenik baskılar kentsel ve kırsal alanlarda büyük zararlar oluşturmaktadır (Yılmaz vd., 2019; Winkler vd., 2021). Bunlar arasında aşırı otlatma, erozyonla toprak kaybı, orman yangınları, arazi örtüsü ve arazi kullanımı değişimi, doğadaki bitki örtüsünün yanlış amaçlarla aşırı derecede kullanımı biyoçeşitliliği tehdit etmekte, birçok endemik ve nadir bitki türü yok olmaktadır (Gülçin ve Deniz, 2020). Bu durum ekosistemin tahrip olmasına neden olmakta ve doğal yaşam dengesi bozulmaktadır.

Türkiye doğal bitki örtüsü bakımından dünyanın en zengin ülkelerinden biridir. Bunun nedeni Akdeniz, İran-Turan ve Avrupa-Sibirya fitocoğrafik bölgelerinin Anadolu'da bulunması ve bitki topluklarının yer yer birbiri ile ilişki içinde olmasıdır (Davis, 1965). Ayrıca iklim farklılıkları, topoğrafik çeşitlilik, jeolojik ve jeomorfolojik çeşitlilik, deniz, göl, akarsu gibi değişik su ortamları çeşitliliği, 0-5000 m'ler arasında değişen yükseklik farklılıkları, üç değişik bitki coğrafyası bölgesinin birleştiği bir yerde oluşu, Anadolu'nun doğusu ve batısı arasında ekolojik farklılıklarının bulunması ve bütün bu ekolojik çeşitliliğin floristik çeşitliliğe yansıması (Türkmen, 1987) ülkemizin biyoçeşitliliğinin zengin olmasının en önemli göstergesidir (Ergüner vd., 2019). Günümüzde bu zengin potansiyelin tespiti ve korunmasına yönelik bilimsel çalışmalar giderek önem kazanmış ve peyzaj mimarlığı uygulamalarında doğal bitki türlerinden yararlanma çalışmaları hız kazanmıştır.

Peyzaj planlama çalışmalarının en önemli elementlerinden birisi olan bitkiler; çiçek, meyve, dal, yaprak renk ve şekilleri, mevsimsel renk değişimleri ve doku özellikleri ile kullanıldıkları mekânlara estetik değerler katmaktadır. Buna ek olarak, bitkilerin; hava kirliliğini önleme, gürültüyü maskeleme, rüzgâr, toz ve gaz etkilerini azaltma, kent formuna dinamik etki verme, ulaşım akslarını belirleme, erozyonu önleme, iklim koşullarını iyileştirme gibi fonksiyonel özellikleri ile de yaşam kalitesini arttırmaktadırlar (Kurşun, 2014). Özellikle bazı odunsu türler, mevsimsel döngü içerisinde farklı renklenmeleri ile görsel hareketlilik ve estetik güzellik sağlamaları yanında meyve özellikleri nedeniyle canlıların besin döngüleri, iklim faktörleri ve hava döngüsü süreçleri üzerine yaptığı katkılarla ekolojik dengeyi koruyarak sürdürülebilirliği sağladıkları için gerek kentsel gerekse kırsal peyzaj çalışmalarında tasarım bitkisi olarak kullanılmaktadırlar (Yaşar ve Düzgüneş, 2013).

Ülkemizde genellikle peyzaj tasarımı uygulamalarında bitkisel materyalin yurt dışından ithal edildiği ve bu bitkilerin de ülkemizin iklim koşullarına uyum sağlayamadığı göz önüne alındığında, yeşil alan düzenlemelerinde doğal bitki türlerinden yararlanmanın gereği ve öneminin büyük olduğu görülmektedir. Doğal bitki türleri ile araştırmaların sınırlı olması nedeniyle bu çalışmada Sivas ilinde doğal olarak yetişen odunsu ve çalı türlerinin, peyzaj mimarlığı açısından estetik ve fonksiyonel özellikleri değerlendirilmiştir. Bu değerlendirmede, öncelikle daha önce farklı alanlarda ve benzer konularda yapılmış olan çalışmalar incelenmiştir.

Bitki türlerinin incelendiği çalışmalar; bölge, havza veya kent ölçeğinde yürütülmüştür. Örneğin; Tortum Çayı havzasında tespit edilen doğal odunsu türlerin peyzaj mimarlığında kullanım olanakları incelendiğinde estetik ve fonksiyonel katkıları olduğu vurgulanırken (Irmak ve Y1lmaz, 2008), ErzurumUzundere karayolu boyunca belirlenen alanlarda tespit edilen doğal odunsu türlerin özellikle karayolunda yönlendiriciliğe katkısı olduğu belirtilmiştir (Yılmaz ve Yılmaz, 2009). Aynı şekilde Ekici 
(2010) yaptığ1 çalışmada Bartın kenti ve yakın çevresinde belirlediği doğal bitki örtüsünün peyzaj tasarımlarında kullanılabileceğini vurgularken, Aslan ve Akan (2019) Şanlıurfa çevresindeki orman, park ve bahçelerde tespit edilen doğal odunsu türlerin peyzaj mimarlığı açısından önemini belirtmiştir. Ayrıca Kılıç vd. (2016) de çalışmalarında meyve özellikli odunsu bitki türlerinin peyzaj amaçlı bitkisel tasarımlarda kullanılabileceğini vurgulamışlardır.

Sonuç olarak bu çalışmada elde edilen bilgiler doğrultusunda Sivas ilinde peyzaj mimarlığında kullanılabilecek doğal odunsu ve çalı türleri belirlenmiş ve bu türlerin korunması ve çoğaltılmasına yönelik öneriler geliştirilmiştir.

\section{Materyal ve Yöntem}

Araştırma materyalini Sivas ili ve ilde doğal olarak yetişen odunsu ve çalı türleri oluşturmaktadır. Sivas ili, İç Anadolu Bölgesi’nin yüzölçümü bakımından Konya ilinden sonra ikinci büyük ilidir (Şekil 1).

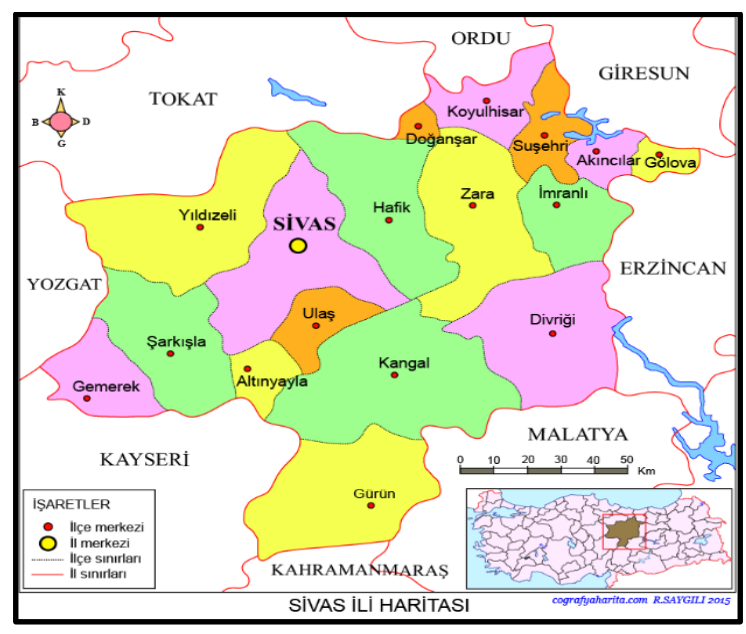

Şekil 1. Sivas ilinin konumu (Anonim, 2021)

İlin büyük bir kısmı yüksek platolar ve dağlık alanlardan oluşmaktadır. Alanda ovalar oldukça azdır ve genellikle vadilerin genişlemesiyle oluşmuştur. İl sınırları içerisinde Gemerek-Şarkışla, YıldızeliSuşehri ovaları bulunmaktadır. İlin en önemli dağları il kuzeyinde yer almakta olup kuzey sınırı ile Kızılırmak arasında kalmaktadır. Başlıcaları: Yıldız Dağı (2537 m.), Kızıldağ (3015 m.), Hezanlı Dağı (2283 m.), Gövdeli Dağı (2719 m.) ve Bey Dağı (2802 m.)'dır (Sivas Valiliği, 2002). Araştırma alanının toprak örtüsü; ormanların büyük ölçüde tahribe uğradığı ve stepe dönüştüğü sahalarda kahverengi topraklar, step-orman geçiş sahalarında kestane renkli topraklar (Mollisol), kuru ve yarı nemli orman sahalarında kahverengi orman toprakları ve geniş yataklı akarsu vadilerinin (Kızılırmak ve kolları vb.) taşkın ovalarında alüvyal topraklardan oluşmaktadır (Atalay, 1994). Sivas ili akarsular yönünden de zengin bir ildir. Türkiye'nin dört büyük ırmağından olan Kızılırmak, Yeşilırmak, Fırat Nehri, Ceyhan Nehri'nin kollarından bazıları kaynağını il sınırları içerisinden almaktadır. Seyhan Nehri'nin kollarından biri olan Zamantı Çayı'da kaynağını il sınırları içerisinden alır (Akpulat ve Karakuş, 2019). Sivas il sınırı içerisinde büyük göller yoktur (Şekil 2). Fakat çok sayıda küçük göl bulunmaktadır. Bunlardan bazıları; Hafik, Lota, Tödürge ve Gökpınar gölleridir. İlin büyük bir kesimi yazları sıcak ve kurak, kışları soğuk ve karlı geçen karasal İç Anadolu ikliminin etkisi altındadır. Alan karasal iklim kuşağında yer aldığı için genellikle bozkır bitkileri (Astragalus spp., Verbascum spp., Thymus spp.) hakimdir (Şekil 2). 

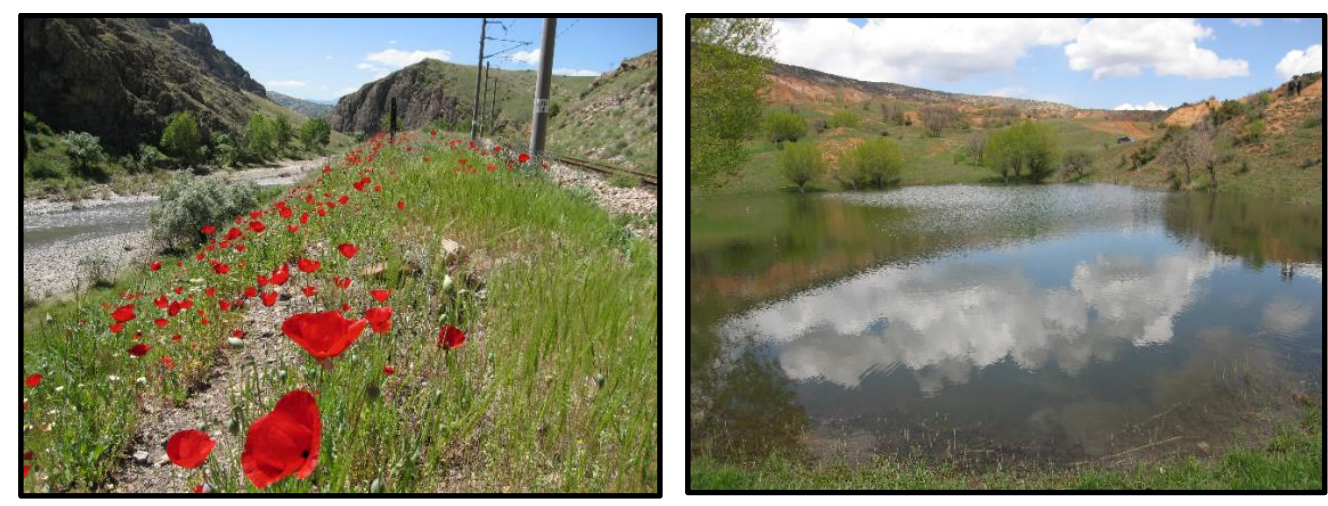

Şekil 2. Sivas ili Divriği ilçesi doğal bitki örtüsü ile akarsu ve küçük göl oluşumlarına birer örnek (Bozkurt, 2019)

İl endemik bitki türleri (Achillea sintenisii Hub.-Mor., Centaurea sivasica Wag., Cousinia sivasica Hub.-Mor., Astragalus ulashensis Hub.-Mor., Salvia vermifolia Hedge et Hub.-Mor., Helichrysum noeanum Boiss.) ve yeni tespit edilen türler açısından da son derece zengindir. Bu konuda araştırma yapan Şahin (2014) "Hezanlı dağları ve çevresinin (Gürün-Sivas) vejetasyonu" adlı çalışmasında alanda 27 yeni bitki türü saptamıştır.

$\mathrm{Bu}$ araştırma kapsamında kullanılan yöntem ise arazi çalışması, bitkilerin teşhisi ve büro çalışmalarından oluşmaktadır. Bu nedenle çalışmada ilk olarak Sivas özelinde yapılan flora çalışmaları incelenmiş ve bu çalışmalardan ilin odunsu ve çalı türlerinin listesi çıkartılmıştır. Daha sonra bu listede yer alan türler 2019 yılının vejetasyon döneminde (Nisan-Haziran) ilin bazı bölgelerine (Sivas-Gürün arası, Divriği, Şarkışla-Gürün arası, Beydağı ve Hezanlı Dağları) birer hafta ara ile gidilerek yerinde gözlenmiş ve fotoğraflar çekilmiştir. Bu araştırmalar sırasında bitkilerin fonksiyonel özellikleri (erozyon önleme, perdeleme, karayolu kenarı ve refüj bitkilendirmesi, maden alanı yeniden ağaçlandırma, kaya bahçesi kullanımı, su kıyıları ve kıyı koruma) ve estetik özellikleri (çiçek güzelliği, yaprak güzelliği, meyve etkisi, form güzelliği, gövde güzelliği, gölge etkisi, sonbaharda yaprak renkliliği, kış etkisi) tespit edilmiştir. Alanda tespit edilen türlerin teşhisinde ve bu bitkilerin peyzaj mimarlığında kullanım olanaklarının belirlenmesinde çeşitli araştırmalardan yararlanılmıştır. Bu araştırmalar; Davis (19651988), Polunin (1981), Dönmez (1998), Karakuş (2009), Şahin (2014), Bozkurt (2016), Bozkurt ve Akkemik (2019), Irmak ve Yılmaz (2008), Kılıç vd. (2016), Yılmaz ve Yılmaz (2009), Ekici (2010), Korkut (1993) ve Yılmaz (2006)'dır. Sonuç olarak elde edilen veriler doğrultusunda çalışmada Sivas'ta bulunan odunsu ve çalı türlerinin sahip olduğu estetik ve fonksiyonel özellikler incelenerek bu türlerin peyzaj mimarlığında kullanım olanakları belirlenmiştir.

\section{Bulgular}

$\mathrm{Bu}$ araştırmada, bitki türlerine dayalı bulguların doğallık değerlendirmeleri çalışmalarında kullanılabilecek önemli fonksiyonları olduğu görülmüştür. Bulgulara göre, 21 familyaya ait toplam 56 odunsu ve çalı takson tespit edilmiştir. Bu taksonlara ait yetişme yerleri, endemizm ve element durumu ile peyzaj mimarlığında kullanımlarına yönelik fonksiyonel ve estetik özellikleri Tablo 1'de verilmiştir. Buna göre alanda tespit edilen doğal odunsu ve çalı taksonlarından 2 tanesi endemik olup 10'u İranTuran, 6's1 Avrupa-Sibirya, 1'i ise Doğu Akdeniz elementidir.

Sivas dağlık ve engebeli bir arazi yapısına sahip olduğu için erozyon önlemede kullanılabilecek bitkiler ön planda yer almaktadır. Bu nedenle kök yapısı kuvvetli kayalık ve kalkerli zeminlerde kolaylıkla yetişebilen türler erozyonu önlemede de etkili olmaktadır. Bu amaçla alanda tespit edilen odunsu türlerden erozyonu önlemede kullanılabilecek taksonlar; Pinus sylvestris L., Juniperus communis L., 
Juniperus excelsa Bieb., Juniperus sabina L., Quercus robur L., Quercus macranthera Fisch., Quercus pubescens Willd., Fraxinus excelsior L. ssp. excelsior L., Sorbus umbellata (Desf.) var. umbellata (Desf.), Sorbus umbellata (Desf.) var. cretica (Lindl.), Amygdalus orientalis Mill., Hippophae rhamnoides L., Elaeagnus angustifolia L., Celtis glabrata Stev.ex Plan., Rosa spp. ve Crataegus spp.'dir (Tablo 1).

Alanda perdeleme açısından uygun olan taksonlar ise; Juniperus excelsa Bieb., Elaeagnus angustifolia L., Ficus carica L. ssp. rupestris (Hauss.), Ailanthus altissima (Mill.), Rhus coriaria L., Cornus sanguinea L., Frangula alnus Mill. ssp. alnus Mill., Rosa sp., Crataegus sp. gibi formu, dokusu, kök yapısı itibariyle sık yapıda olan bitkilerdir (Tablo 1). Bu bitkilerin rüzgâr ve gürültü perdesi olmasının yanı sıra çit bitkisi olarak da kullanılabileceği belirlenmiştir. Aslanboğa (2002), yaptığı çalışmada buna benzer bitkileri sınırlama, perdeleme ve mekân oluşturma için önermiştir.

Karayolu kenarı ve refüj bitkilendirmesinde kullanılabilecek taksonlar; Juniperus communis L., Juniperus excelsa Bieb., Juniperus sabina L., Sambucus nigra L., Amygdalus orientalis Mill., Berberis vulgaris L., Berberis crataegina DC., Ailanthus altissima Mill., Ephedra major Host., Ficus carica L. ssp. rupestris Hauss., Hippophea rhamnoides L., Cornus sanguinea L., Frangula alnus Mill. ssp. alnus Mill., Rhamnus oleoides L. ssp. graecus Boiss. et Reut., Celtis glabrata Stev.ex Plan., Atrophaxis billardieri Jaub. et Spach var. billardieri Jaub. et Spach, Cotaneaster spp., Crataegus spp., ve Rosa spp., olarak belirlenmiştir (Tablo 1). Bu bitkilerin seçilmesinde Tanrıverdi (1987)'nin “orta refüjlerde kullanılacak bitkilerde; karşıdan gelen araçların far 1şıklarını engelleyecek şekilde yerden itibaren dallanabilen, darbeye, toza ve kirliliğe dayanıklı bitkilerin kullanılması gerektiğì" görüşü esas alınmıştır. Aynı şekilde karayolu şev stabilizasyonunda da Ailanthus altissima Mill., Rosa canina L., gibi bitki türlerinin kullanılabileceği önerilmiştir.

Alanda birçok taş ve maden ocağı bulunmaktadır. Bu alanların daha sonra doğal türlerle rehabilite edilmesi önemli bir konudur. Bu amaçla kullanılabilecek taksonlar; Pinus sylvestris L., Juniperus communis L., Juniperus excelsa Bieb., Juniperus sabina L., Hippophae rhamnoides L., Elaeagnus angustifolia L., Ficus carica L. ssp. rupestris Hauss., Celtis glabrata Stev.ex Plan., Ephedra major Host., Ailanthus altissima Mill., Fraxinus excelsior L. ssp. excelsior L., Rosa spp., Crataegus spp., Sorbus spp.'dir (Tablo 1). Maden ve taş ocakları ile çöp alanlarının 1slahında Bayraktar (1980), Ürgenç (2000) ve Köse vd (1993) yaptıkları çalışmalarda Ephedra major Host., Rosa canina L., Ailanthus altissima (Mill.) ve Hippophea rhamnoides L. gibi türleri önermişlerdir.

Doğal park ve bahçelerin tasarımında doğal görünümleri nedeniyle kaya bahçeleri büyük öneme sahiptirler. Kaya bahçelerinin en önemli bitkisel elemanları küçük ağaç ve çalı grubu bitkilerdir. Çalışma alanı genellikle kayalık alanlardan oluştuğu için bu alanlarda doğal olarak yetişebilen bitkilerden estetik özellikleri açısından öne çıkan bazı bitkilerin kaya bahçelerinde kullanımları uygun bulunmuştur. $\mathrm{Bu}$ taksonlar; Krascheninnikovia ceratoides L., Cerasus hippophaeoides Bornm., Berberis vulgaris L., Berberis crataegina DC., Amygdalus orientalis Mill., Ephedra major Host., Cotaneaster integerrimus Medik., Cotaneaster nummularia Fisch. et Mey., Crataegus tanacetifolia Lam. Pers., Cerasus incana (Pall.) Spach var. incana (Pall.) Spach, Atrophaxis billardieri Jaub. et Spach var. billardieri Jaub. et Spach, Daphne oleoides Schreber ssp. oleoides Schreber, Daphne oleoides Schreber ssp. kurdica Bornm., Rhamnus oleoides L. ssp. graecus Boiss. et Reut., Juniperus sabina L.,'dir (Tablo 1). Ürgenç (1998) yaptığı çalışmada Berberis spp., Cotoneaster spp. ve Juniperus spp. gibi türlerin kaya bahçelerinde kullanılmasının uygun olduğunu belirtmiştir.

Alanda tespit edilen türlerden su kıyılarının bitkilendirilmesi ve kıyı koruma çalışmalarında kullanılabilecek bitkiler arasında genellikle suyu seven ve yüksek taban suyu seviyesine sahip alanlarda 
doğal olarak yetişen Populus nigra L., Populus alba L., Salix alba L. ve Salix excelsa J. F. Gmelin, Ailanthus altissima (Mill.), Fraxinus excelsior L. ssp. excelsior L., Rubus canescens DC., Rubus caesius L. gibi taksonların kullanılabileceği belirlenmiştir (Tablo 1).

Araştırma alanında tespit edilen bitkilerin çiçek, yaprak, form güzelliği, meyve, gölge etkisi, sonbaharda yaprak renkliliği ve kış manzarası oluşturabilme gibi özelliklerine bakılarak estetik özellikleri belirlenmiştir. Bu özelliklere sahip olan bitkilerden estetik özellikler açısından ön plana çıkan bazı taksonlar şunlardır; Pinus sylvestris L., Juniperus communis L., Juniperus excelsa Bieb., Juniperus sabina L., Prunus spinosa L. ssp. dasyphylla (Schur) Domin, Prunus divaricata Ledeb. ssp. divaricata Ledeb., Sorbus umbellata (Desf.) var. umbellata (Desf.), Sorbus umbellata (Desf.) var. cretica (Lindl.), Pyrus syriaca Boiss., Pyrus elaeagnifolia Pallas ssp. elaeagnifolia Pallas, Cerasus incana (Pallas) Spach var. incana (Pallas) Spach, Cerasus mahaleb (L.) Miller var. mahaleb (L.) Miller, Hippophae rhamnoides L., Elaeagnus angustifolia L., Morus nigra L., Ficus carica L. ssp. rupestris (Hauss.), Quercus robur L., Quercus macranthera Fisch., Quercus pubescens Willd., Salix alba L., Salix excelsa . F. Gmelin, Populus nigra L., Populus alba L., Berberis vulgaris L., Berberis crataegina DC., Juglans regia L., Ailanthus altissima (Mill.), Rhus coriaria L., Sambucus nigra L., Cornus sanguinea L., Frangula alnus Mill. ssp. alnus Mill., Celtis glabrata Stev.ex Plan., Fraxinus excelsior L. ssp. excelsior L., Rosa spp., Crataegus spp.'dir (Tablo 1). Bu bitkilerin estetik özelliklerinden dolay1 kentsel mekânlarda (parklar, endüstriyel bölgeler, kamu ve özel bahçeler ile karayolu şev ve refüjlerinde) yapılan düzenlemelerde soliter veya gruplar halinde kullanılabilecekleri belirlenmiştir. Sonuç olarak araştırma alanında tespit edilen ve doğal olarak yetişen bu türler estetik ve fonksiyonel özellikleri nedeniyle kentsel ve kırsal peyzaj tasarımlarında kullanılabilirler. Bu taksonlardan bazılarına ilişkin fotoğraflar Şekil 2'de verilmiştir. 
Tablo 1. Sivas'ta doğal olarak yetişen bazı odunsu ve çalı türlerinin peyzaj mimarlı̆̆ında kullanım olanakları

\begin{tabular}{|c|c|c|c|c|c|c|c|c|c|c|c|c|c|c|c|c|c|c|}
\hline \multirow[t]{2}{*}{ Familya } & \multirow[t]{2}{*}{ Tür Adı } & \multirow[t]{2}{*}{ Yetiştiği yer } & \multirow[b]{2}{*}{ 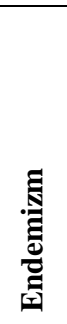 } & \multirow[b]{2}{*}{ 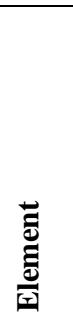 } & \multicolumn{6}{|c|}{ Fonksiyonel özellikler } & \multicolumn{8}{|c|}{ Estetik özellikler } \\
\hline & & & & & 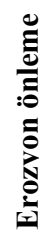 & 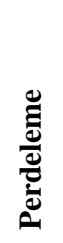 & 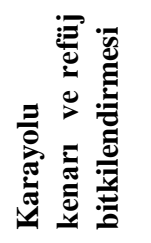 & 豞 & 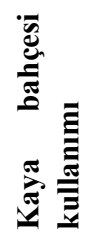 & 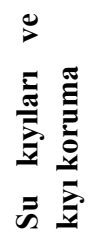 & 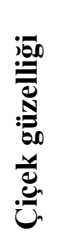 & 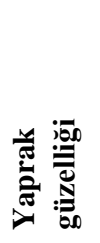 & 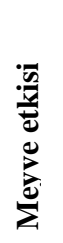 & 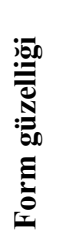 & 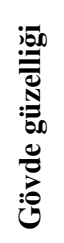 & 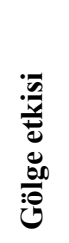 & 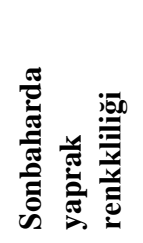 & 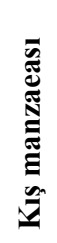 \\
\hline PINACEAE & Pinus sylvestris $\mathrm{L}$. & $\begin{array}{l}\text { Sivas-Suşehri- } \\
\text { Y1ldizeli }\end{array}$ & & & $\sqrt{ }$ & & & $\sqrt{ }$ & & & & $\sqrt{ }$ & & $\sqrt{ }$ & $\sqrt{ }$ & $\sqrt{ }$ & & $\sqrt{ }$ \\
\hline CUPRASSACEAE & Juniperus communis L. & $\begin{array}{l}\text { Sivas-Suşehri- } \\
\text { Zara }\end{array}$ & & & $\sqrt{ }$ & & $\sqrt{ }$ & $\sqrt{ }$ & & & & $\sqrt{ }$ & $\sqrt{ }$ & $\sqrt{ }$ & $\sqrt{ }$ & & & $\sqrt{ }$ \\
\hline$"$ & Juniperus excelsa Bieb. & Sivas-Gürün & & & $\sqrt{ }$ & $\sqrt{ }$ & $\sqrt{ }$ & $\sqrt{ }$ & & & & $\sqrt{ }$ & $\sqrt{ }$ & $\sqrt{ }$ & $\sqrt{ }$ & $\sqrt{ }$ & & $\sqrt{ }$ \\
\hline$"$ & Juniperus sabina L. & $\begin{array}{l}\text { Sivas-Yıldız } \\
\text { dağları }\end{array}$ & & & $\sqrt{ }$ & & $\sqrt{ }$ & $\sqrt{ }$ & $\sqrt{ }$ & & & $\sqrt{ }$ & $\sqrt{ }$ & $\sqrt{ }$ & $\sqrt{ }$ & & & $\sqrt{ }$ \\
\hline $\begin{array}{l}\text { CHENOPODIACE } \\
A E\end{array}$ & $\begin{array}{l}\text { Krascheninnikovia ceratoides } \\
\text { (L.) Güldenst. }\end{array}$ & Sivas-Gürün & & & $\sqrt{ }$ & & & & $\sqrt{ }$ & & & & & $\sqrt{ }$ & & & & \\
\hline ROSACEAE & $\begin{array}{lll}\begin{array}{l}\text { Prunus spinosa } \\
\text { dasyphylla }\end{array} & \text { L. } & \text { ssp. } \\
\end{array}$ & Sivas-Tatlıçok & & & & & & & & & $\sqrt{ }$ & $\sqrt{ }$ & $\sqrt{ }$ & $\sqrt{ }$ & $\sqrt{ }$ & $\sqrt{ }$ & & \\
\hline$"$ & $\begin{array}{l}\text { Prunus divaricata ssp. } \\
\text { divaricata (Schur) Domin. }\end{array}$ & Sivas & & & & & & & & & $\sqrt{ }$ & $\sqrt{ }$ & $\sqrt{ }$ & $\sqrt{ }$ & $\sqrt{ }$ & $\sqrt{ }$ & & \\
\hline " & $\begin{array}{l}\text { Cerasus } \quad \text { hippophaeoides } \\
\text { Bornm. }\end{array}$ & $\begin{array}{l}\text { Sivas-Divriği- } \\
\text { Dumluca Dağ1 }\end{array}$ & $\begin{array}{l}\mathbf{E} \\
\mathbf{N}\end{array}$ & İ-T & & & $\sqrt{ }$ & & $\sqrt{ }$ & & $\sqrt{ }$ & $\sqrt{ }$ & $\sqrt{ }$ & $\sqrt{ }$ & & & & \\
\hline$"$ & Rubus canescens DC. & Sivas-Gürün & & A-S & & & & & & $\sqrt{ }$ & $\sqrt{ }$ & $\sqrt{ }$ & $\sqrt{ }$ & $\sqrt{ }$ & & & & \\
\hline$"$ & Rubus caesius L. & Sivas-Gürün & & & & & & & & $\sqrt{ }$ & $\sqrt{ }$ & $\sqrt{ }$ & $\sqrt{ }$ & $\sqrt{ }$ & & & & \\
\hline$"$ & Rosa hemisphaerica J. Herrm. & Sivas & & $\dot{\mathbf{I}}-\mathbf{T}$ & $\sqrt{ }$ & $\sqrt{ }$ & $\sqrt{ }$ & $\sqrt{ }$ & & & $\sqrt{ }$ & $\sqrt{ }$ & $\sqrt{ }$ & $\sqrt{ }$ & $\sqrt{ }$ & & & \\
\hline " & Rosa villosa $\mathrm{L}$. & $\begin{array}{l}\text { Sivas-Yıldız } \\
\text { Dağları }\end{array}$ & & & $\sqrt{ }$ & $\sqrt{1}$ & $\sqrt{ }$ & $\sqrt{ }$ & & & $\sqrt{ }$ & $\sqrt{ }$ & $\sqrt{ }$ & $\sqrt{ }$ & $\sqrt{ }$ & & & \\
\hline " & Rosa jundzillii Besser & $\begin{array}{l}\text { Sivas-Yıldız } \\
\text { Dağları }\end{array}$ & & $\mathbf{A - S}$ & $\sqrt{ }$ & $\sqrt{ }$ & $\sqrt{ }$ & $\sqrt{ }$ & & & $\sqrt{ }$ & $\sqrt{ }$ & $\sqrt{ }$ & $\sqrt{ }$ & $\sqrt{ }$ & & & \\
\hline$"$ & Rosa pulverulenta Bieb. & Sivas-Gürün & & & $\sqrt{ }$ & $\sqrt{ }$ & $\sqrt{ }$ & $\sqrt{ }$ & & & $\sqrt{ }$ & $\sqrt{ }$ & $\sqrt{ }$ & $\sqrt{ }$ & $\sqrt{ }$ & & & \\
\hline " & Rosa montana Chaix & Sivas- Divriği & & & $\sqrt{ }$ & $\sqrt{ }$ & $\sqrt{ }$ & $\sqrt{ }$ & & & $\sqrt{ }$ & $\sqrt{ }$ & $\sqrt{ }$ & $\sqrt{ }$ & $\sqrt{ }$ & & & \\
\hline " & Rosa canina L. & Sivas- Karaçayır & & & $\sqrt{ }$ & $\sqrt{ }$ & $\sqrt{ }$ & $\sqrt{ }$ & & & $\sqrt{ }$ & $\sqrt{ }$ & $\sqrt{ }$ & $\sqrt{ }$ & $\sqrt{ }$ & & & \\
\hline$"$ & Rosa foetida J. Herrm. & Sivas-Gürün & & & $\sqrt{ }$ & $\sqrt{ }$ & $\sqrt{ }$ & $\sqrt{ }$ & & & $\sqrt{ }$ & $\sqrt{ }$ & $\sqrt{ }$ & $\sqrt{ }$ & $\sqrt{ }$ & & & \\
\hline " & $\begin{array}{l}\text { Rosa heckeliana Tratt. ssp. } \\
\text { orientalis Meikle }\end{array}$ & Sivas-Gürün & & $\dot{\mathbf{I}}-\mathbf{T}$ & $\sqrt{ }$ & $\sqrt{ }$ & $\sqrt{1}$ & $\sqrt{ }$ & & & $\sqrt{ }$ & $\sqrt{ }$ & $\sqrt{ }$ & $\sqrt{ }$ & $\sqrt{ }$ & & & \\
\hline " & $\begin{array}{l}\text { Cotaneaster integerrimus } \\
\text { Medik. }\end{array}$ & $\begin{array}{l}\text { Sivas-Beydağı- } \\
\text { Zara }\end{array}$ & & & & & $\sqrt{ }$ & & $\sqrt{ }$ & & $\sqrt{ }$ & $\sqrt{ }$ & $\sqrt{ }$ & & & & & \\
\hline
\end{tabular}


Tablo 1.Sivas’ta doğal olarak yetişen bazı odunsu ve çalı türlerinin peyzaj mimarlığında kullanım olanakları (Devamı)

\begin{tabular}{|c|c|c|c|c|c|c|c|c|c|c|c|c|c|c|c|c|c|c|}
\hline \multirow[t]{2}{*}{ Familya } & \multirow[t]{2}{*}{ Tür Adı } & \multirow[t]{2}{*}{ Yetiştiği yer } & \multirow{2}{*}{\multicolumn{2}{|c|}{ 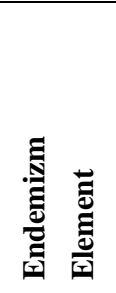 }} & \multicolumn{6}{|c|}{ Fonksiyonel özellikler } & \multicolumn{8}{|c|}{ Estetik özellikler } \\
\hline & & & & & 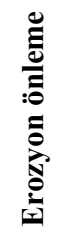 & 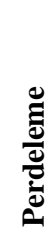 & 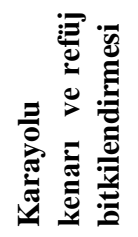 & 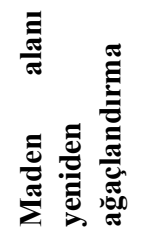 & 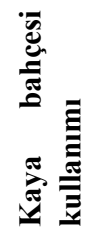 & 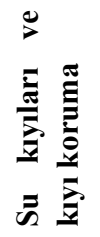 & 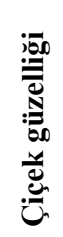 & 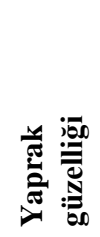 & 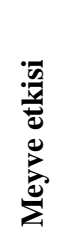 & 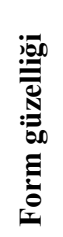 & 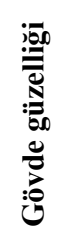 & 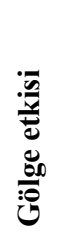 & 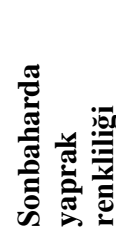 & 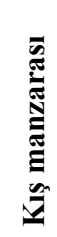 \\
\hline ROSACEAE & Cotaneaster nummularia Fisch. et Mey. & Sivas-Ulaş & & & & & $\sqrt{ }$ & & $\sqrt{ }$ & & $\sqrt{ }$ & $\sqrt{ }$ & $\sqrt{ }$ & $\sqrt{ }$ & & & & \\
\hline " & Crataegus tanacetifolia (Lam.) Pers. & Sivas-Suşehri & & & $\sqrt{ }$ & $\sqrt{ }$ & $\sqrt{ }$ & $\sqrt{ }$ & & & $\sqrt{ }$ & $\sqrt{ }$ & $\sqrt{ }$ & $\sqrt{ }$ & $\sqrt{ }$ & & & $\sqrt{ }$ \\
\hline " & $\begin{array}{l}\text { Crataegus orientalis Pallas ex M. Bieb. } \\
\text { var. orientalis Pallas ex M. Bieb. }\end{array}$ & Sivas & & & $\sqrt{ }$ & $\sqrt{ }$ & $\sqrt{ }$ & $\sqrt{ }$ & & & $\sqrt{ }$ & $\sqrt{ }$ & $\sqrt{ }$ & $\sqrt{ }$ & $\sqrt{ }$ & & & $\sqrt{ }$ \\
\hline " & Crataegus meyeri Pojark. & $\begin{array}{l}\text { Sivas-Suşehri- } \\
\text { Divriği }\end{array}$ & & $\begin{array}{l}\mathbf{I}-\mathbf{T} \\
\end{array}$ & $\sqrt{ }$ & $\sqrt{ }$ & $\sqrt{ }$ & $\sqrt{ }$ & & & $\sqrt{ }$ & $\sqrt{ }$ & $\sqrt{ }$ & $\sqrt{ }$ & $\sqrt{ }$ & & & $\sqrt{ }$ \\
\hline " & $\begin{array}{l}\text { Sorbus umbellata (Desf.) Fritsch var. } \\
\text { umbellata (Desf.) Fritsch }\end{array}$ & Sivas-Gürün & & & $\sqrt{ }$ & & & $\sqrt{ }$ & & & $\sqrt{ }$ & $\sqrt{ }$ & $\sqrt{ }$ & $\sqrt{ }$ & $\sqrt{ }$ & $\sqrt{ }$ & & \\
\hline$"$ & $\begin{array}{l}\text { Sorbus umbellata (Desf.) Fritsch var. } \\
\text { cretica (Lindl.) Schneider }\end{array}$ & Sivas-Suşehri & & & $\sqrt{ }$ & & & $\sqrt{ }$ & & & $\sqrt{ }$ & $\sqrt{ }$ & $\sqrt{ }$ & $\sqrt{ }$ & $\sqrt{ }$ & $\sqrt{ }$ & & \\
\hline " & Pyrus syriaca Boiss. & Sivas-Alacahan & $\begin{array}{l}\mathbf{E} \\
\mathbf{N}\end{array}$ & & & & & & & & $\sqrt{ }$ & & $\sqrt{ }$ & $\sqrt{ }$ & $\sqrt{ }$ & $\sqrt{ }$ & & \\
\hline " & $\begin{array}{llll}\begin{array}{l}\text { Pyrus elaeagnifolia } \\
\text { elaeagnifolia } \text { Pallas }\end{array} & \text { Pallas } & \text { ssp. } \\
\end{array}$ & $\begin{array}{l}\text { Sivas-Yıldız } \\
\text { Dağları-Tatlıca }\end{array}$ & & & & & & & & & $\sqrt{ }$ & & $\sqrt{ }$ & $\sqrt{ }$ & $\sqrt{ }$ & $\sqrt{ }$ & & \\
\hline$"$ & Amygdalus orientalis Miller & Sivas-Gürün & & $\overline{\mathrm{I}}-\mathbf{T}$ & $\sqrt{ }$ & & $\sqrt{ }$ & & $\sqrt{ }$ & & $\sqrt{ }$ & & $\sqrt{ }$ & $\sqrt{ }$ & & & & \\
\hline " & $\begin{array}{l}\text { Cerasus incana (Pallas) Spach var. } \\
\text { incana (Pallas) Spach }\end{array}$ & Sivas-Gürün & & $\overline{\mathrm{I}}-\mathbf{T}$ & & & $\sqrt{ }$ & & $\sqrt{ }$ & & $\sqrt{ }$ & $\sqrt{ }$ & $\sqrt{ }$ & $\sqrt{ }$ & $\sqrt{ }$ & & & \\
\hline$"$ & $\begin{array}{llll}\text { Cerasus mahaleb } & \text { (L.) Miller var. } \\
\text { mahaleb (L.) Miller } & & & \\
\end{array}$ & Sivas-Gürün & & & & & & & & & $\sqrt{ }$ & $\sqrt{ }$ & $\sqrt{ }$ & $\sqrt{ }$ & $\sqrt{ }$ & $\sqrt{ }$ & & \\
\hline $\begin{array}{l}\text { THYMELAEAC } \\
\text { EAE }\end{array}$ & $\begin{array}{l}\text { Daphne oleoides Schreber ssp. oleoides } \\
\text { Schreber }\end{array}$ & $\begin{array}{l}\begin{array}{l}\text { Sivas- } \\
\text { dağ1-Gürün }\end{array} \\
\text { Yıldız }\end{array}$ & & & & & & & $\sqrt{ }$ & & $\sqrt{ }$ & $\sqrt{ }$ & $\sqrt{ }$ & $\sqrt{ }$ & & & & \\
\hline$"$ & $\begin{array}{l}\text { Daphne oleoides Schreber ssp. kurdica } \\
\text { Bornm. }\end{array}$ & $\begin{array}{l}\text { Sivas-Gürün- } \\
\text { Gökpınar }\end{array}$ & & $\dot{\mathrm{I}}-\mathbf{T}$ & & & & & $\sqrt{ }$ & & $\sqrt{ }$ & $\sqrt{ }$ & $\sqrt{ }$ & $\sqrt{ }$ & & & & \\
\hline $\begin{array}{l}\text { ELAEAGNACE } \\
A E\end{array}$ & Hippophae rhamnoides $\mathrm{L}$. & Sivas-Alacahan & & & $\sqrt{ }$ & & & $\sqrt{ }$ & & & $\sqrt{ }$ & $\sqrt{ }$ & $\sqrt{ }$ & $\sqrt{ }$ & $\sqrt{ }$ & $\sqrt{ }$ & & \\
\hline$"$ & Elaeagnus angustifolia $\mathrm{L}$. & Sivas-Gürün & & & $\sqrt{ }$ & $\sqrt{ }$ & & $\sqrt{ }$ & & & $\sqrt{ }$ & $\sqrt{ }$ & $\sqrt{ }$ & $\sqrt{ }$ & $\sqrt{ }$ & & & \\
\hline MORACEAE & Morus nigra $\mathrm{L}$. & Sivas- Gürün & & & & & & & & & & $\sqrt{ }$ & $\sqrt{ }$ & $\sqrt{ }$ & $\sqrt{ }$ & $\sqrt{ }$ & & \\
\hline$"$ & Ficus carica L. ssp. rupestris Browicz. & Sivas- Gürün & & $\begin{array}{l}\mathbf{I}-\mathbf{T} \\
\end{array}$ & & $\sqrt{ }$ & & $\sqrt{ }$ & & & & $\sqrt{ }$ & $\sqrt{ }$ & $\sqrt{ }$ & $\sqrt{ }$ & $\sqrt{ }$ & $\sqrt{ }$ & \\
\hline FAGACEAE & Quercus robur L. & Sivas-Şarkışla & & & $\sqrt{ }$ & & & & & & & $\sqrt{ }$ & $\sqrt{ }$ & $\sqrt{ }$ & $\sqrt{ }$ & $\sqrt{ }$ & $\sqrt{ }$ & $\sqrt{ }$ \\
\hline
\end{tabular}


Tablo 1.Sivas’ta doğal olarak yetişen bazı odunsu ve çalı türlerinin peyzaj mimarlığında kullanım olanakları (Devamı)

\begin{tabular}{|c|c|c|c|c|c|c|c|c|c|c|c|c|c|c|c|c|c|c|}
\hline \multirow[t]{2}{*}{ Familya } & \multirow[t]{2}{*}{ Tür Adı } & \multirow{2}{*}{$\begin{array}{l}\text { Yetiştiği } \\
\text { yer }\end{array}$} & \multirow[b]{2}{*}{ 気 } & \multirow[b]{2}{*}{$\begin{array}{l}\overrightarrow{\vec{U}} \\
\stackrel{\vec{U}}{x}\end{array}$} & \multicolumn{6}{|c|}{ Fonksiyonel özellikler } & \multicolumn{8}{|c|}{ Estetik özellikler } \\
\hline & & & & & 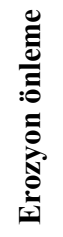 & 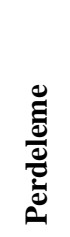 & 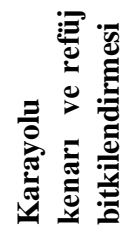 & 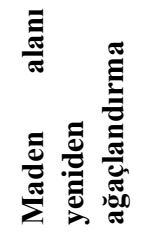 & 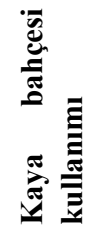 & 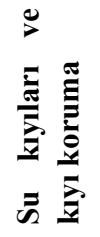 & 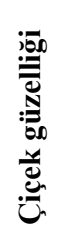 & 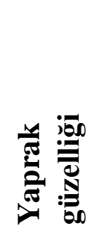 & 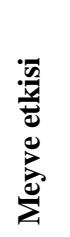 & 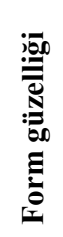 & 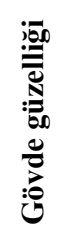 & 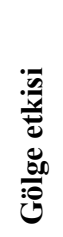 & 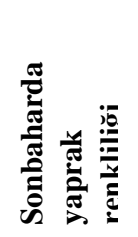 & 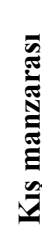 \\
\hline FAGACEAE & Quercus macranthera Fisch. et Mey. & $\begin{array}{l}\text { Sivas-Y1ldız } \\
\text { dağ1 }\end{array}$ & & & $\sqrt{ }$ & & & & & & & $\sqrt{ }$ & $\sqrt{ }$ & $\sqrt{ }$ & $\sqrt{ }$ & $\sqrt{ }$ & $\sqrt{ }$ & $\sqrt{ }$ \\
\hline$"$ & Quercus pubescens Willd. & $\begin{array}{l}\text { Sivas- } \\
\text { Y1ldızeli } \\
\end{array}$ & & & $\sqrt{ }$ & & & & & & & $\sqrt{ }$ & $\sqrt{ }$ & $\sqrt{ }$ & $\sqrt{ }$ & $\sqrt{ }$ & $\sqrt{ }$ & $\sqrt{ }$ \\
\hline SALICACEAE & Salix alba $\mathrm{L}$. & Sivas-Gürün & & A-S & & & & & & $\sqrt{ }$ & & $\sqrt{ }$ & & $\sqrt{ }$ & $\sqrt{ }$ & $\sqrt{ }$ & $\sqrt{ }$ & $\sqrt{ }$ \\
\hline$"$ & Salix excelsa J. F. Gmelin & Sivas-Gürün & & İ-T & & & & & & $\sqrt{ }$ & & $\sqrt{ }$ & & $\sqrt{ }$ & $\sqrt{ }$ & $\sqrt{ }$ & $\sqrt{ }$ & $\sqrt{ }$ \\
\hline$"$ & Populus nigra $\mathrm{L}$. & Sivas-Gürün & & & & & & & & $\sqrt{ }$ & & $\sqrt{ }$ & & $\sqrt{ }$ & $\sqrt{ }$ & $\sqrt{ }$ & $\sqrt{ }$ & $\sqrt{ }$ \\
\hline$"$ & Populus alba $\mathrm{L}$. & Sivas-Gürün & & & & & & & & $\sqrt{ }$ & & $\sqrt{ }$ & & $\sqrt{ }$ & $\sqrt{ }$ & $\sqrt{ }$ & $\sqrt{ }$ & $\sqrt{ }$ \\
\hline BERBERIDACEAE & Berberis vulgaris $\mathrm{L}$. & Sivas-Gürün & & & & & $\sqrt{ }$ & & $\sqrt{ }$ & & $\sqrt{ }$ & $\sqrt{ }$ & $\sqrt{ }$ & $\sqrt{ }$ & $\sqrt{ }$ & & & \\
\hline$"$ & Berberis crataegina DC. & Sivas-Gürün & & İ-T? & & & $\sqrt{ }$ & & $\sqrt{ }$ & & $\sqrt{ }$ & $\sqrt{ }$ & $\sqrt{ }$ & $\sqrt{ }$ & $\sqrt{ }$ & & & \\
\hline JUGLANDACEAE & Juglans regia $\mathrm{L}$. & Sivas-Gürün & & & & & & & & & & $\sqrt{1}$ & $\sqrt{ }$ & $\sqrt{1}$ & $\sqrt{1}$ & $\sqrt{1}$ & $\sqrt{1}$ & $\sqrt{1}$ \\
\hline SIMAROUBACEAE & Ailanthus altissima (Miller) Swingle & Sivas-Gürün & & & & $\sqrt{ }$ & $\sqrt{ }$ & $\sqrt{ }$ & & $\sqrt{ }$ & $\sqrt{ }$ & $\sqrt{ }$ & & $\sqrt{ }$ & $\sqrt{ }$ & $\sqrt{ }$ & $\sqrt{ }$ & $\sqrt{ }$ \\
\hline ANACARDIACEAE & Rhus coriaria $\mathrm{L}$. & Sivas-Gürün & & & & $\sqrt{ }$ & & & & & & $\sqrt{ }$ & $\sqrt{ }$ & $\sqrt{ }$ & $\sqrt{ }$ & & $\sqrt{ }$ & $\sqrt{ }$ \\
\hline EPHEDRACEAE & Ephedra major Host & Sivas-Gürün & & & & & $\sqrt{ }$ & $\sqrt{ }$ & $\sqrt{ }$ & & & $\sqrt{ }$ & $\sqrt{ }$ & $\sqrt{ }$ & $\sqrt{ }$ & & & \\
\hline CAPRIFOLIACEAE & Sambucus nigra L. & Sivas-Gürün & & A-S & & & $\sqrt{1}$ & & & & $\sqrt{ }$ & $\sqrt{1}$ & $\sqrt{ }$ & $\sqrt{1}$ & $\sqrt{ }$ & $\sqrt{ }$ & $\sqrt{1}$ & \\
\hline CORNACEAE & Cornus sanguinea $\mathrm{L}$. & Sivas-Gürün & & A-S & & $\sqrt{ }$ & $\sqrt{ }$ & & & & $\sqrt{ }$ & $\sqrt{ }$ & $\sqrt{ }$ & $\sqrt{ }$ & $\sqrt{ }$ & & $\sqrt{ }$ & $\sqrt{ }$ \\
\hline RHAMNACEAE & $\begin{array}{l}\text { Frangula alnus Miller ssp. alnus } \\
\text { Miller }\end{array}$ & Sivas-Gürün & & A-S & & $\sqrt{ }$ & $\sqrt{ }$ & & & & $\sqrt{ }$ & $\sqrt{ }$ & $\sqrt{ }$ & $\sqrt{ }$ & $\sqrt{ }$ & & $\sqrt{ }$ & \\
\hline$"$ & $\begin{array}{l}\text { Rhamnus oleoides L. ssp. graecus } \\
\text { (Boiss. et Reut.) Holmb. }\end{array}$ & Sivas-Gürün & & D.A & & & $\sqrt{ }$ & & $\sqrt{ }$ & & $\sqrt{ }$ & $\sqrt{ }$ & $\sqrt{ }$ & $\sqrt{ }$ & & & & \\
\hline ULMACEAE & Celtis glabrata Steven ex Planc. & Sivas-Gürün & & & $\sqrt{ }$ & $\sqrt{ }$ & $\sqrt{ }$ & $\sqrt{ }$ & & & $\sqrt{ }$ & $\sqrt{ }$ & $\sqrt{ }$ & $\sqrt{ }$ & $\sqrt{ }$ & & $\sqrt{ }$ & \\
\hline OLEACEAE & Fraxinus excelsior L. ssp. excelsior L. & Sivas-Gürün & & & $\sqrt{ }$ & & & $\sqrt{ }$ & & $\sqrt{ }$ & $\sqrt{ }$ & $\sqrt{ }$ & & $\sqrt{1}$ & $\sqrt{ }$ & $\sqrt{ }$ & $\sqrt{ }$ & $\sqrt{ }$ \\
\hline POLYGONACEAE & $\begin{array}{l}\text { Atrophaxis billardieri Jaub. et Spach } \\
\text { var. billardieri Jaub. et Spach }\end{array}$ & Sivas-Gürün & & $\dot{\mathbf{I}}-\mathbf{T}$ & & & $\sqrt{ }$ & & $\sqrt{ }$ & & $\sqrt{ }$ & $\sqrt{ }$ & $\sqrt{ }$ & $\sqrt{ }$ & & & & \\
\hline $\begin{array}{l}\text { EN: Endemik } \\
\text { I-T: İran-Turan }\end{array}$ & & & & & & D.A: & $\begin{array}{l}\text { vrupa-Si } \\
\text { oğu Akd }\end{array}$ & & & & & & & & & & & \\
\hline
\end{tabular}




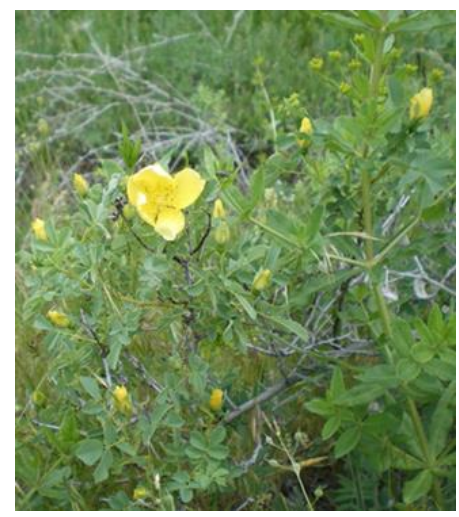

Rosa foetida J. Herrm. (Bozkurt, 2019)

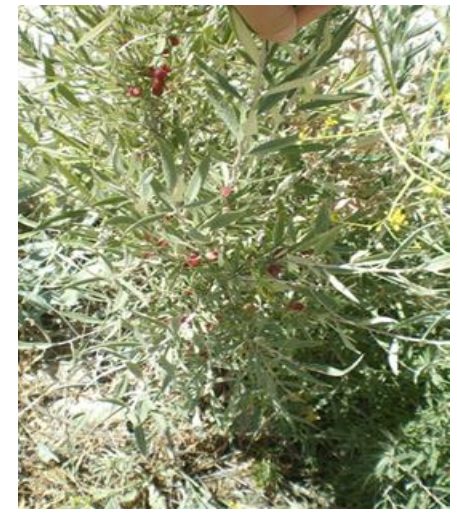

Cerasus incana (Pallas) Spach var. incana (Pallas) Spach (Bozkurt, 2019)

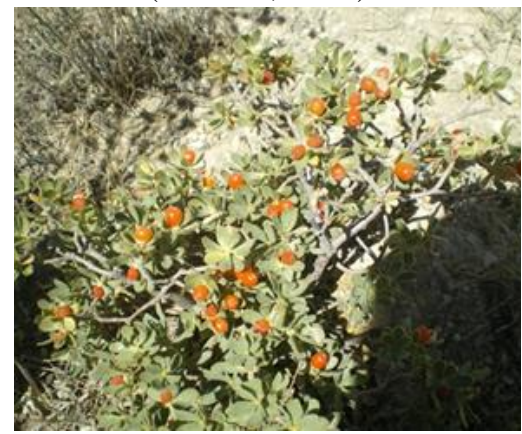

Daphne oleoides Schreber ssp. oleoides Schreber (Bozkurt, 2016)

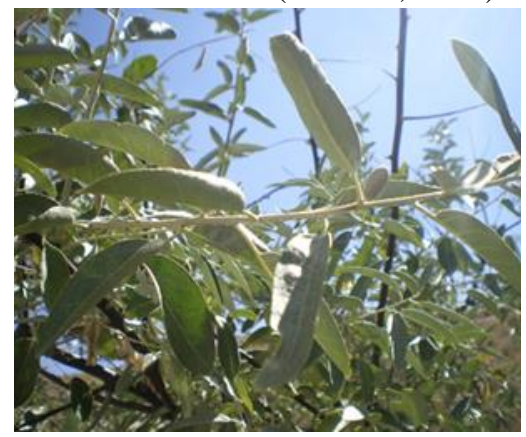

Elaeagnus angustifolia L. (Bozkurt, 2019)

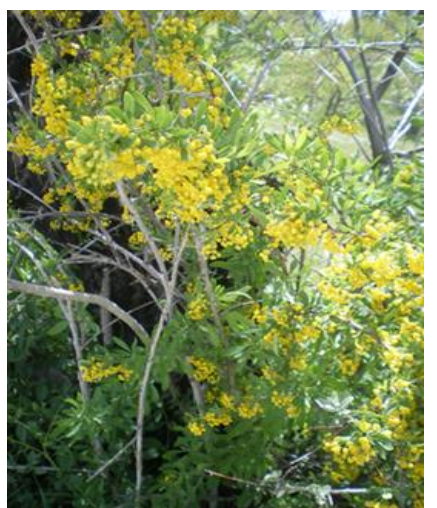

Berberis vulgaris L. (Bozkurt, 2019)

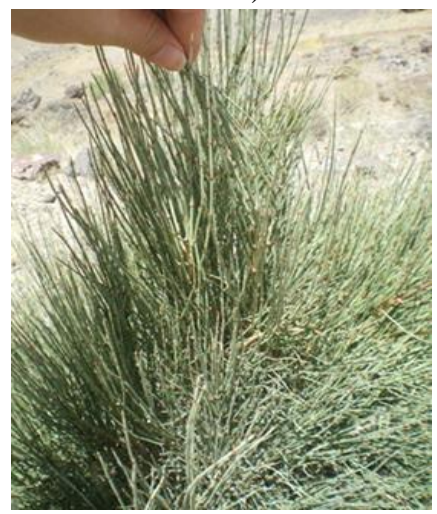

Ephedra major Host (Bozkurt, 2016)

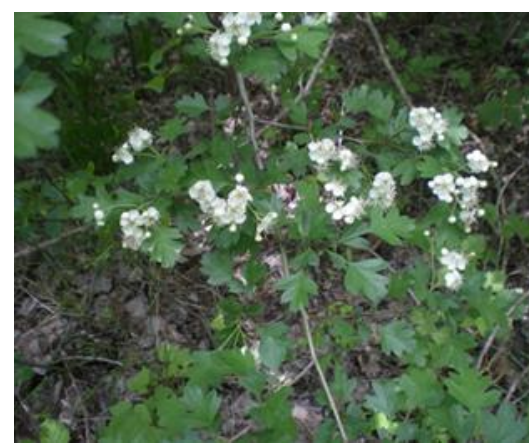

Rubus caesius L. (Bozkurt, 2019)

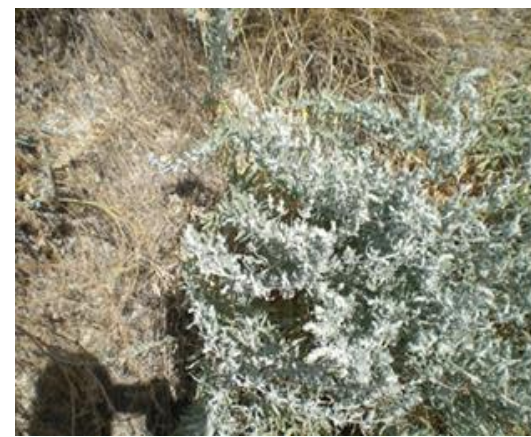

Amygdalus orientalis Miller (Bozkurt, 2019)

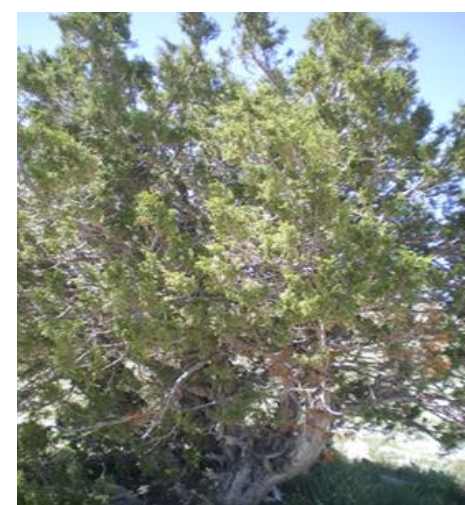

Juniperus excelsa M. Bieb.

(Bozkurt, 2019)

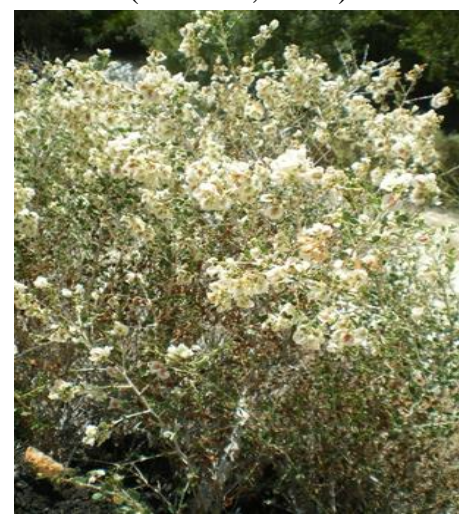

Atrophaxis billardieri Jaub. et Spach var. billardieri Jaub. et Spach (Bozkurt, 2016)

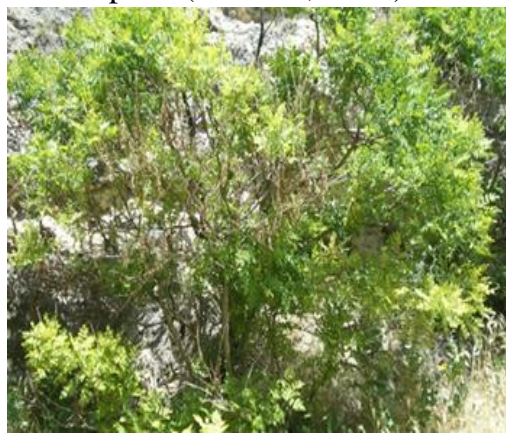

Rhus coriaria L. (Bozkurt, 2019)

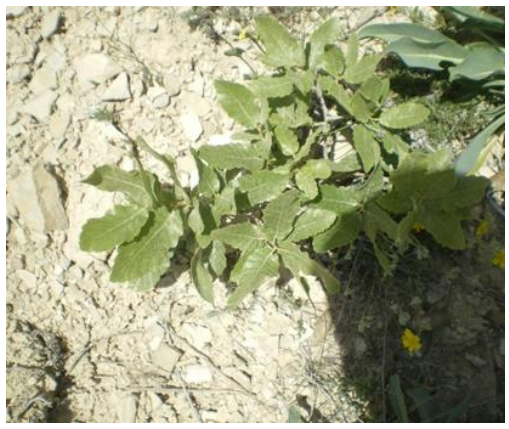

Quercus robur L. (Bozkurt, 2019)

Şekil 2. Sivas'ta doğal olarak yetişen ve peyzaj tasarımlarında kullanılabilecek bazı odunsu ve çalı türleri 


\section{Tartışma ve Sonuç}

Coğrafi konumu ve iklim özellikleri nedeniyle Sivas doğal bitki çeşitliliği açısından oldukça zengindir. Ancak bu zengin kaynaktan peyzaj tasarım ve uygulamalarında yeterince yararlanılmamaktadır. Özellikle kamu kurum bahçelerinde, parklarda, yol ve refüj düzenlemelerinde büyük oranda yabancı orjinli bitkiler kullanılmakta, bozulan doğal peyzajın rehabilite edilmesinde bile ithal bitkiler kullanılmaktadır. Bu durum o bölgenin doğal bitki örtüsü ile uyumsuz bir görüntü oluşturmasının yanı sıra çoğunlukla ithal bitkilerin de bölgeye uyum sağlayamayıp ölmesi ile sonuçlanmaktadır. Aynı zamanda alanda doğal bitki örtüsüne yönelik üretim çabaları da çok yetersiz kalmaktadır. Bu nedenle bu tür çalışmaların peyzaj tasarım ve uygulamalarında doğal türlerin kullanımını artıracağı öngörülmektedir. Sivas özelinde yapılan bu çalışmada peyzaj mimarlığı çalışmalarında kullanılabilecek 21 familyaya ait toplam 56 odunsu ve çalı taksonu tespit edilmiş ve bu taksonların estetik ve fonksiyonel özellikleri açısından peyzaj tasarım ve uygulamalarında kullanılabilecekleri belirlenmiştir. Ayrıca bu çalışma ile Sivas'ın coğrafi konumu, iklim özellikleri, jeomorfolojik yapısı göz önüne alındığında, alanda bulunan doğal odunsu ve çalı türlerinin önemli bir çeşitliliğe sahip olduğu ortaya konulmuştur.

Ülkemizin coğrafi koşulları göz önüne alındığında benzer koşullara sahip kentlerde peyzaj uygulamalarında bu türlerin çoğaltılması, değerlendirilmeye alınması büyük önem arz etmektedir. Ayrıca bu bitkiler kültüre alınarak, botanik bahçelerinde tanıtılmalı ve sergilenmeli, alanın flora tanıtımına yönelik kitap, broşür ve kataloglar hazırlanmalıdır. Fidanlıklarda bu bitkilerin satılması teşvik edilmeli ve üretilmesine yönelik faaliyetler desteklenmelidir. Dijital ortamda da alanın flora zenginliğini içeren web sayfaları oluşturularak, yöre ve bölge halkına bu türler tanıtılmalı ve hangi amaçlarla kullanabileceklerinin bilgisi verilmelidir.

\section{Kaynaklar}

Akpulat, H. A., Karakuş, C. B. (2019). Sivas ilinde yayılış gösteren endemik türlerin tehlike kategorilerine göre mekansal dağılımı, VIII. Umteb International Congress on Vocatıonal \& Technical Sciences, Sivas-Turkey. Erişim adresi: https://www.researchgate.net/publication/340273786

Aslan, M., Akan, H. (2019). A study of natural woody plants of forest in Şanlıurfa-determination of detection and landscape values of parks and garden plants, Biological Diversity and Conservation, 12/1 (2019) 50-65, DOI: 10.5505/biodicon.2019.43433. Erişim adresi: https://www.researchgate.net/publication/332983823

Aslanboğa, İ. (2002). Odunsu bitkilerle bitkilendirmenin işleve uygun tasarımının, uygulanmasının ve bakımının planlanmasl, TC. Orman Bakanlığı, Ege Ormancılık Araştırma Müdürlüğ̈̈, S: 100. İzmir.

Atalay, İ. (1994). Türkiye Vejetasyon Coğrafyası, Ege Üniversitesi Basımevi, İzmir.

Bayraktar, A. (1980). İzmir ve çevresi yeşil örtüsünde bazı doğal bitki türlerinin saptanması ve peyzaj çalışmalarında kullanım olanakları üzerinde araştırmalar, Ege Üniversitesi Ziraat Fakültesi Peyzaj Mimarlığ Süs Bitkileri Bölümü, Türkiye Peyzaj Mimarisi Derneği Yayınları No:1980/2, İzmir.

Bozkurt, S. G. (2016). Gürün (Sivas) ilçe merkezi biyotoplarının özellikleri ve haritalanması üzerine araştırmalar (Doktora Tezi). Erişim adresi: https://tez.yok.gov.tr/UlusalTezMerkezi

Bozkurt, S. G., Akkemik Ü. (2019). Gürün (Sivas) ilçesinde tespit edilen doğal odunsu bitkilerin peyzaj mimarlığı açısından kullanım olanakları, Journal of Forestry, 15 (2) , 137-152. Erişim adresi: https://dergipark.org.tr/tr/pub/duzceod/issue/51465/393027 
EJFS - Sivas'da doğal olarak yetişen bazı odunsu ve çalı türlerinin peyzaj mimarlığında kullanım olanaklarının belirlenmesi-Bozkurt 2021

Davis, P. H. (1965-1988). Flora of Turkey and The East Aegean Islands. Edinburgh University Press,, Edinburgh Vol. 1-10.

Dönmez, E. (1998). Gövdeli Dă̆ı (Sivas-Kayseri) florası (Doktora Tezi). Erişim adresi: https://tez.yok.gov.tr/UlusalTezMerkezi

Ekici, B. (2010). Bartın kenti ve yakın çevresinde yetişen bazı doğal bitkilerin kentsel mekanlarda kullanım olanakları, Süleyman Demirel Üniversitesi Orman Fakültesi Dergisi, Seri: A, Sayı: 2, S: 110-126. Erişim adresi: https://dergipark.org.tr/tr/download/article-file/195752

Ergüner, Y., Kumar, J., Hoffman, F. M., Dalfes, H. N., Hargrove, W. W. (2019). Mapping ecoregions under climate change: a case study from the biological 'crossroads' of three continents, Turkey. Landscape Ecology, 34(1), 35-50.

Gülçin, D., \& Deniz, B. (2020). Remote sensing and GIS-based forest fire risk zone mapping: The case of Manisa, Turkey. Turkish Journal of Forestry, 21(1), 15-24.

Irmak, M. A., Yılmaz, H. (2008). Tortum Çayı Havzası'nın odunsu bitkilerinin peyzaj mimarlığı açısından fonksiyonel ve estetik amaçlı kullanım olanaklarının belirlenmesi, Biyolojik Çesitlilik ve Koruma, 1(1): 1-12. Erişim adresi: https://arastirmax.com/en/publication/biyolojij-cesitlilik-koruma/1/1/tortum-cayi-havzasinin$\underline{\text { odunsu }}$

Karakuş, Ş., (2009). Tohma vadisi (Gürün-Darende) florası (Yüksek Lisans Tezi). Erişim adresi: https://tez.yok.gov.tr/UlusalTezMerkezi

Kılıç T., Kazaz, S., Ergür, G. E., Gül, A., (2016). Meyve özellikli odunsu bitki türlerinin peyzaj amaçlı bitkisel tasarımda kullanılabilme olanakları, VI. Süs Bitkileri Kongresi, 19-22 Nisan 2016, Antalya. Erişim adresi: http://www.sonerkazaz.com/wp-content/uploads/6_u.pdf

Korkut, A. B., (1993). Trakya bölgesi doğal bitki örtüsünde peyzaj planlama çalışmaları yönünden değerlendirilebilecek bazı bitkisel materyalin saptanması. Doğa Turkish Journal of Agriculture and Forestry, 17 (1), 315-330.

Köse, H., Şimşir, F., Güney, A., (1993). Açık maden işletmelerinde rekültivasyon ve rekreasyon, Dokuz Eylül Üniversitesi Mühendislik Fakültesi Yayınları, No:236, İzmir.

Kurşun, H. (2014). Peyzaj mimarlığı çalışmalarında süs bitkisi olarak kullanılan meyve türleri (Mezuniyet Tezi). Ankara Üniversitesi Ziraat Fakültesi, Bahçe Bitkileri Bölümü.

Polunin, O., (1969). Flowers of Europe Flowers, Oxford University Press, Oxford.

Sivas Valiliğii, 2002. Sivas 2002 İl Yıllı̆̆ı. Sivas

Şahin, B., (2014). Hezanlı dağları ve çevresinin (Gürün/Sivas) vejetasyonu. (Doktora Tezi). Erişim adresi: https://tez.yok.gov.tr/UlusalTezMerkezi

Tanrıverdi, F. (1987). Peyzaj mimarlı̆̆ı bahçe sanatının temel ilkeleri ve uygulama metodları. Atatürk Üniversitesi Ziraat Fakültesi, Yayın No: 291, S:367, Erzurum.

Türkmen, N. (1987). Çukurova Üniversitesi kampus alanının doğal bitkileri, hayat formları ve habitatları. (Yüksek Lisans Tezi). Erişim adresi: https://tez.yok.gov.tr/UlusalTezMerkezi

Ürgenç, S., İ. (2000). Kırsal Peyzaj, YTÜ. Mimarlık Fakültesi Şehir ve Bölge Planlama Bölümü. S:173-188. YTÜ Basım Yayın Merkezi, İstanbul.

Winkler, K., Fuchs, R., Rounsevell, M., Herold, M. (2021). Global land use changes are four times greater than previously estimated. Nature communications, 12(1), 1-10. 
EJFS - Sivas'da doğal olarak yetişen bazı odunsu ve çalı türlerinin peyzaj mimarlığında kullanım olanaklarının belirlenmesi-Bozkurt 2021

Yaşar, Y., Düzgüneş, E. (2013). Peyzaj tasarımına sürdürülebilirlik kavramının entegrasyonu: bir stüdyo çalışması, Inönü Üniversitesi Sanat ve Tasarım Dergisi, 3 (7): 31-43. Erişim adresi: $\underline{\text { https://dergipark.org.tr/tr/download/article-file/92474 }}$

Yılmaz, H., (2006). Erzurum-Uzundere Karayolu Şevlerinde Doğal Olarak Yetişen Bitkilerin Estetik ve Fonksiyonel Yönden Değerlendirilmesi (Yüksek Lisans Tezi). Erişim adresi: https://tez.yok.gov.tr/UlusalTezMerkezi

Yılmaz, H., Yılmaz, H. (2009). Karayolu şevlerinde doğal olarak yetişen odunsu bitkilerin kullanım alanlarının irdelenmesi; Erzurum-Uzundere örneği, Süleyman Demirel Üniversitesi Orman Fakültesi Dergisi, Seri: A, Say1: 1, S: 101-111. Erişim adresi: https://dergipark.org.tr/tr/pub/tjf/issue/20892/224320

Yılmaz, K. T., Alphan, H., Gülçin, D. (2019). Assessing degree of landscape naturalness in a Mediterranean coastal environment threatened by human activities. Journal of Urban Planning and Development, 145(2), 05019004.

Anonim, 2021: https://www.milliyet.com.tr/egitim/haritalar/sivas-haritasi-sivas-ilceleri-nelerdir-sivas-ilininnufusu-kactir-kac-ilcesi-vardir-6306591 [Erişim tarihi: 14-05-2021]. 